University of Nebraska - Lincoln

DigitalCommons@University of Nebraska - Lincoln

Faculty Publications, Department of Physics and Astronomy

Research Papers in Physics and Astronomy

2002

\title{
Phase and Absorption Gratings for Electrons
}

\author{
Hong Gao \\ University of Nebraska - Lincoln \\ Glen Gronniger \\ University of Nebraska - Lincoln \\ Daniel Friemund \\ University of Nebraska - Lincoln \\ Alex Cronin \\ Massachusetts Institute of Technology, cronin@physics.arizona.edu
}

Follow this and additional works at: https://digitalcommons.unl.edu/physicsfacpub

Part of the Atomic, Molecular and Optical Physics Commons, and the Quantum Physics Commons

Gao, Hong; Gronniger, Glen; Friemund, Daniel; and Cronin, Alex, "Phase and Absorption Gratings for Electrons" (2002). Faculty Publications, Department of Physics and Astronomy. 126.

https://digitalcommons.unl.edu/physicsfacpub/126

This Article is brought to you for free and open access by the Research Papers in Physics and Astronomy at DigitalCommons@University of Nebraska - Lincoln. It has been accepted for inclusion in Faculty Publications, Department of Physics and Astronomy by an authorized administrator of DigitalCommons@University of Nebraska Lincoln. 
Published in the Proceedings of the XVIII International Conference on Atomic Physics: The Expanding Frontier of Atomic Physics (2002), p. 133-141. Conference held in Cambridge, Massachusetts, USA, July 28-August 2, 2002. Copyright 2002, World Scientific. Used by permission.

\title{
Phase and Absorption Gratings for Electrons
}

\author{
Hong Gao ${ }^{1}$, Glen Gronniger ${ }^{1}$, Daniel Freimund ${ }^{1}$, Alex Cronin ${ }^{2}$ \\ and Herman Batelaan ${ }^{1}$ \\ ${ }^{1}$ Department of Physics and Astronomy, University of Nebraska-Lincoln, \\ Lincoln, Nebraska, USA \\ ${ }^{2}$ Massachusetts Institute of Technology, Cambridge Massachusetts, USA
}

We report the experimental realization of phase and absorption gratings for electrons. Phase gratings made with standing waves of light with a periodicity of $266 \mathrm{~nm}$ are used to diffract $380 \mathrm{eV}$ electrons [1]. Material gratings of 100 and $200 \mathrm{~nm}$ periodicity are used to diffract $500 \mathrm{eV}$ electrons. We are exploring the possibility to use these gratings for low energy electron interferometry.

\section{Introduction}

In matter optics with atoms many analogies between light and matter waves have been explored. In matter optics with electrons the same does not appear to hold to the same extent. Although electron microscopy in use far supersedes any tool developed in atom optics, this technique is based on only a few optics elements [2]: electron lenses [3], beam splitters (biprisms) [4] and coherent sources [5]. In atom optics, apart from these elements $[6,7,8,9,10]$, mirrors [11], polarizing beam splitters [12], fibers [13], modulators [14], and gratings [15] are a few examples of the many analogies that have been explored. It may be possible to develop some of these techniques for electron optics and find useful applications for them. Here, we would like to present our work on phase and absorption gratings for electrons. For completeness we should mention that absorption gratings for electrons were demonstrated decades ago [16]. The near field diffraction pattern was observed using $50 \mathrm{keV}$ electrons for a grating made from individually deposited wires. We observe the far-fields diffraction pattern at $500 \mathrm{eV}$ for a nano-fabricated grating. Given the ever improving capabilities in nano-fabrication and the variation of patterns that can be made, we feel it is interesting to revisit this field.

\section{Phase gratings}

When electrons pass through a standing light wave, it is possible for the electron wave to diffract from the periodic light structure, just as light 
waves can diffract from the periodic structure of a material grating. This effect, known as the Kapitza-Dirac effect, was proposed in 1933 [17], and was just recently observed [1]. To describe the periodic light structure we can use the analogy with atom optics.

Passing a well-collimated atomic beam through a standing wave laser beam can be described accurately by an atomic plane wave, which accumulates a sinusoidal phase shift as a function of position. The phase shift accumulation by the atomic wave is caused by the interaction, $H_{\mathrm{int}}=-\frac{1}{2} \alpha E^{2}$, between the induced atomic dipole, $d=a E$, and the electric field, $E$, associated with the standing wave, where $a$ is the polarizability. The quadratic dependence on the electric field ensures that the interaction does not average out to zero for the fast oscillating electric field associated with a laser. The spatially modulation of the atomic wave is called a phase grating,

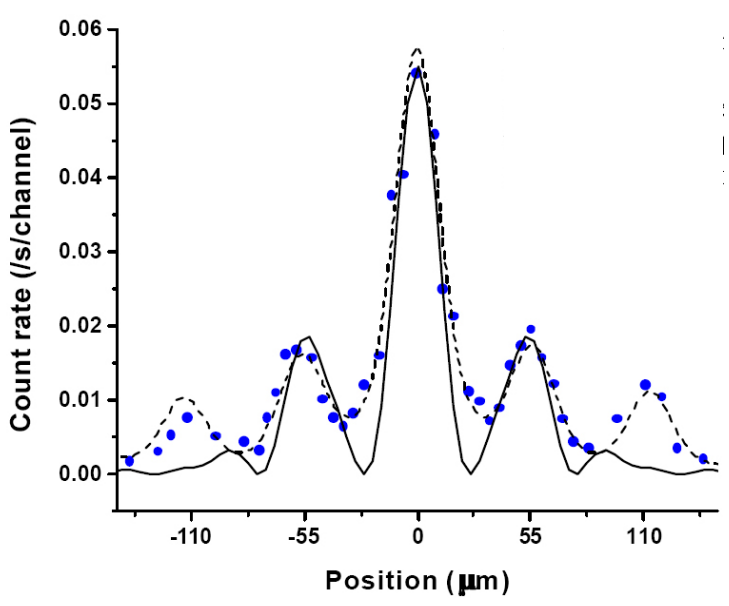

Figure 1: Electrons diffracted from a standing wave of light. Experimental data (dots), numerical integration of the Schrödinger equation (dotted line), and a Fourier transform (solid line).

which can be described in terms of the sinusoidal lightshift potential, $V_{L}=\frac{1}{2} \frac{q^{2} / m}{\omega^{2}-\omega_{0}^{2}} E_{0}^{2} \cos ^{2} k x$

where the classical expression for the atomic polarizability is $\alpha=\frac{q^{2} / m}{\omega_{0}^{2}-\omega^{2}}$ [18].

The passage of an electron through a standing wave of light can be described by the ponderomotive potential, which can be obtained by assuming that the atomic electron is effectively free and $\omega_{0} \rightarrow 0$. This is why it is sometimes stated that the free electron has a resonance at $\omega_{0}=0$. To 
obtain appreciable scattering onewould like the phaseshift, $\varphi \approx E t / \hbar$, to be of the order of one. Using the relation, $I=\frac{1}{2} \varepsilon_{0} c \omega^{2} A_{0}{ }^{2}$, between the laser intensity, $I$, and the vector potential amplitude, $A_{0}$, a straightforward estimation shows that for electrons with energies of hundreds of $\mathrm{eV}$, the laser intensity needed to observe the Kapitza-Dirac effect is about $10^{9}-10^{10} \mathrm{~W} /$ $\mathrm{cm}^{2}$. The simplest calculation to obtain the electron diffraction pattern is made by taking the Fourier transform of the phase modulated plane wave of the incident electron. In figure 1, the measured diffraction pattern (dots) together with a numerical integration of the Schrödinger equation (dotted line) is shown [1]. We have calculated a Fourier transform of the sinusoidally modulated electron wave to give the momentum distribution of the electrons. Starting from a point source the momentum distribution can be propagated to the detector yielding the position distribution (solid line). The Fourier transform is integrated over the spatial (transverse) coherence length of the electron beam. A coherence length of $200 \mathrm{~nm}$ gives results that are in a reasonable agreement with the observed width of the diffraction peaks. The second order peak in the quantum mechanical calculation is much more pronounced due to averaging over various parameters such as the laser beam intensity profile.

Instead of focussing the laser and having a short interaction time, one can use the unfocused laser beams. This will yield an asymmetrical diffraction pattern with predominantly two diffraction peaks instead of a symmetric diffraction pattern. This can only be observed when the incident electron enters the unfocused light beam at the Bragg angle. In this way, one can go from the "thin grating" to the Bragg diffraction regimes. Such a change in behavior can be observed for both atoms [19] and electrons [20]. The implications of using either of these approaches for atomic interferometry have been investigated [21,22].

\section{Absorption gratings}

\subsection{Image charge and gratings}

As an altogether different approach we have explored the use of material gratings. The advantage of using material gratings is higher electron count rates. The laser system used for the experiment briefly described above has a repetition rate of $50 \mathrm{~Hz}$ and a pulse duration of about $10 \mathrm{~ns}$. This means that the laser and the grating are off for most of the time. For the purpose of building an electron interferometer, $\mathrm{CW}$ gratings would increase the count rate by more than $10^{6}$. Perhaps $\mathrm{CO}_{2}$-lasers with lower energy electron beams could be used or build-up cavities might be constructed to meet the intensity requirements. For material gratings we anticipated charging of the grating. In addition image charges would be a concern. To prevent charging, metallic structures were used: one solid gold grating (with a $200 \mathrm{~nm}$ period) and one Silicon Nitride grating, coat- 
ed front and back with gold (with a $100 \mathrm{~nm}$ period). Also of concern was that small amounts of vacuum oil would be adsorbed on the grating and charge up. It turned out that these problems were minor.
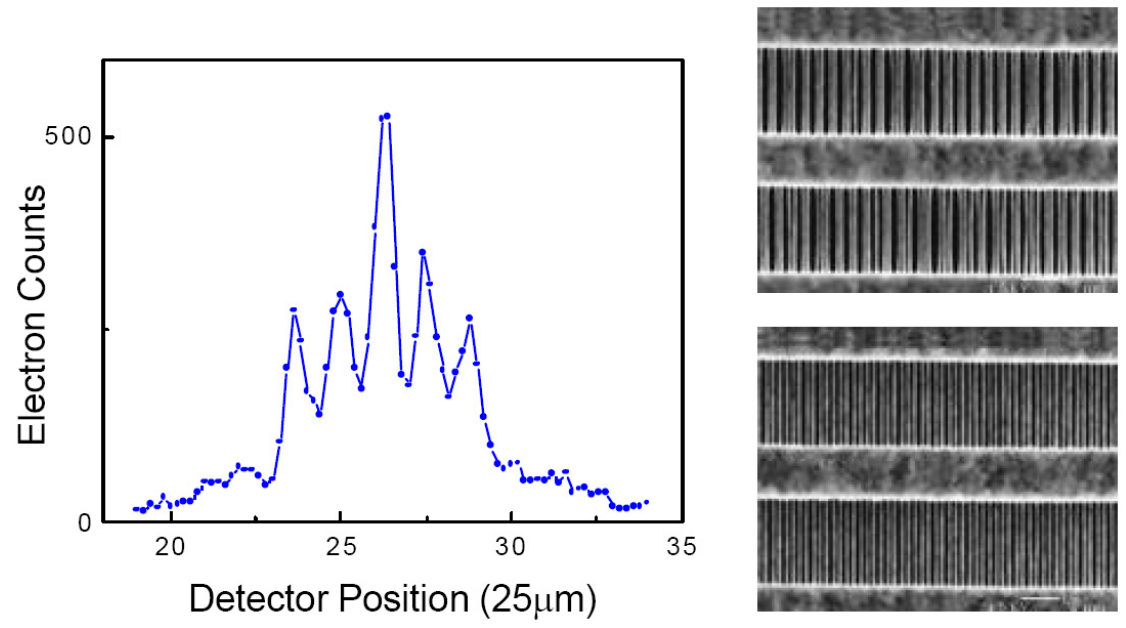

Figure 2: Left: the diffraction pattern of a $500 \mathrm{eV}$ electron beam passing through a material grating with a periodicity of $200 \mathrm{~nm}$. Right: scanning electron microscope pictures of the same grating at two different locations. Part of the grating bars have merged pairwise (and in some places three bars have merged) (top). Part of the grating bars are intact (bottom).

With the first grating, no special alignment was needed to observe a clear diffraction pattern (figure 2). The observed diffraction pattern showed diffraction peaks not only corresponding to a $200 \mathrm{~nm}$ periodicity, but also peaks corresponding to a $400 \mathrm{~nm}$ periodicity (figure 2 left). Scanning electron microscopy pictures of the grating revealed that in parts of the grating the bars had merged pairwise (figure 2 right top), while other parts were intact (figure 2 right bottom). This explains the observed diffraction pattern. To improve the peak resolution and to prevent the merging of the grating bars a gold-coated SiN grating with a periodicity of $100 \mathrm{~nm}$ was used. The diffraction pattern was calculated by Fourier transforming an amplitude modulated wave emerging from the absorption grating. To estimate the influence of the image charge, its phase modulation was multiplied with the amplitude modulated wave and again Fourier transformed. The expected result is a diffraction pattern significantly modified by the image charge (figure 3.). We considered this as a minimum modification of the diffraction pattern, while any additional charging effect might be more severe. However, the experimental data does not show strong evi- 
dence for the effect of the image charge or other charging effects (figure 4). The second order peak in the experimental data is suppressed compared to the third order peak, as is the case for the calculation excluding the image charge effect! We have not systematically addressed this issue yet.

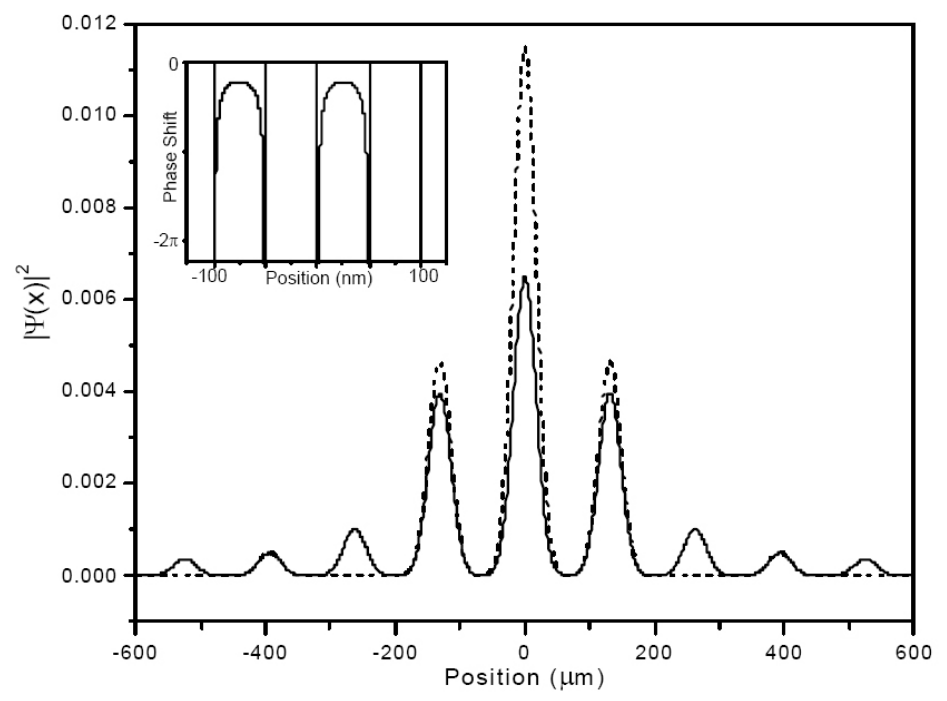

Figure 3: A calculation of the image charge effect on a $500 \mathrm{eV}$ electron beam passing through a grating with a $100 \mathrm{~nm}$ periodicity. Ignoring the image charge, the diffraction pattern is given by the dotted line. Assuming a perfect conductor, the diffraction pattern is modified to the pattern given by the solid line. The inset shows the phase shift as a function of position.

Perhaps it is appropriate to mention that we could not determine if the surface of the grating bars is coated on all sides. So, one might speculate that some of the metal coating is missing so that the image charge could not form. Perhaps a coating is not needed at all. On the other hand, it is also interesting to note that the electrons take about $10^{-14} \mathrm{~s}$ to pass through the grating. One might speculate that the image charge has no time to be formed. This issue needs some further study.

\subsection{Blazed gratings}

It is interesting to be capable to modify the diffraction pattern for the purpose of optimization of Mach-Zehnder interferometers. 


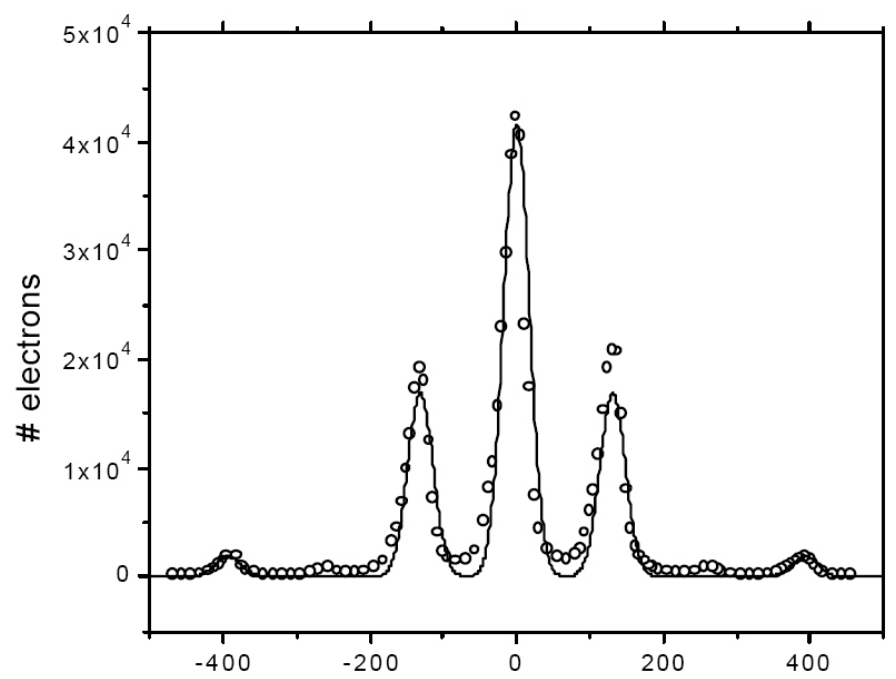

Figure 4: The diffraction pattern of a $500 \mathrm{eV}$ electron beam from a material grating with a periodicity of $100 \mathrm{~nm}$. The solid line is the result of a Fourier transformed periodic electron wave, excluding the effect of image charge.

For phase gratings, one could change the three-peak diffraction pattern into a two-peak Bragg scattering pattern by changing the laser light focus and angle. For optical gratings, the same can be achieved by using blazed reflection gratings. Here we would like to suggest a method to make such a blazed transmission grating for electrons. When one connects the outer edges of the gratings with a voltage, a situation similar to that of a capacitor filled with a series of metal plates exists. The grating bars play the role of the metal plates, while the outer edges of the grating play the role of the capacitor plates. In this case, we would like the grating bars to be coated, but the support structure for the grating bars to be uncoated so that no short circuit between them is present. The potential between each grating line will now drop linearly (in a 1-D model) and make a step from each line to the next. This will cause a phase shift pattern that resembles that of a blazed grating. The phase shift pattern and its resulting diffraction pattern are calculated and presented in figure 5 . It is assumed that $500 \mathrm{eV}$ electrons move through a $100 \mathrm{~nm}$ grating, and that a $25 \mathrm{~V}$ potential difference is placed over a $10 \mu \mathrm{m}$ section of the grating. This will allow our electron beam to pass through the grating, while at the same time keep the necessary voltage within reasonable limits. The obtained diffraction pat- 


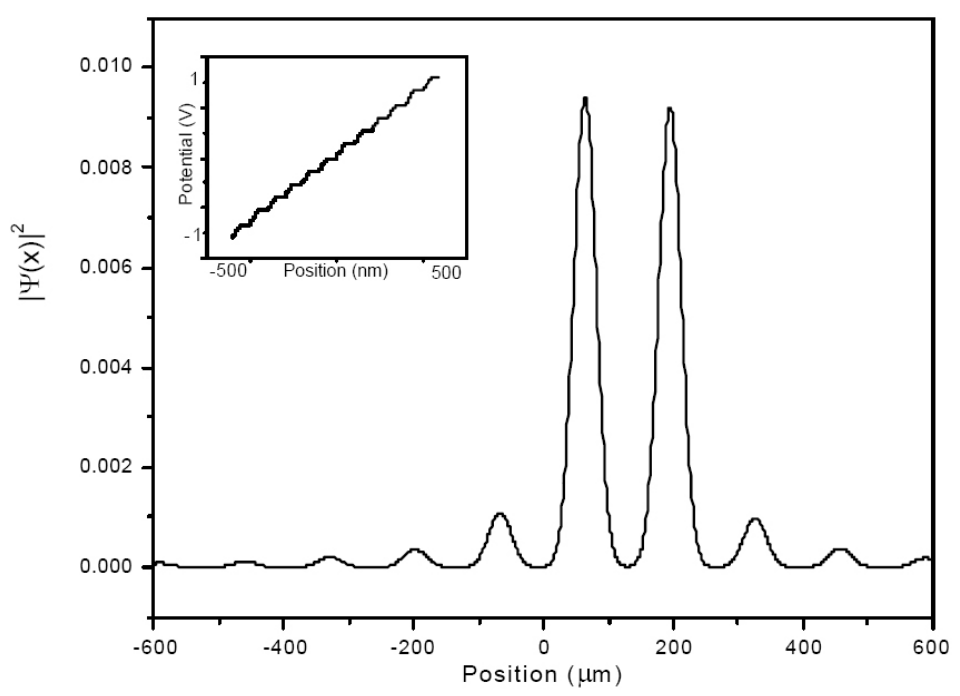

Figure 5: A blazed grating for electrons. The calculated diffraction pattern that may be obtained by placing a voltage difference over a partially coated grating.

tern shows that two peaks are dominant, in analogy to an optical blazed grating.

\subsection{Towards electron interferometry}

One of the straightforward possibilities to construct a low energy electron interferometer is to combine three material gratings to form a MachZehnder interferometer. Before attempting such a device, we looked into the possibility of combining the grating with a biprism. We placed a $12 \mu \mathrm{m}$ gold wire $2.5 \mathrm{~cm}$ behind the material grating. This position was chosen in an attempt to block the zero-order diffraction peak and let the two first order diffraction peaks pass by both sides of the gold wire. When such a combination works appropriately, there are two possible scenarios that follow. Placing a positive voltage on the biprism wire would allow for the recombination of the two electron beams to form a simple interferometer. Placing a negative voltage on the wire would allow the angle between the two coherent electron beams to be increased, which is interesting for large enclosed area interferometers. The wire could be moved laterally with a micrometer to bring it into the correct position. The experimental data is shown in figure 6 . The wire is moved from the left to the right, starting 
from the bottom diffraction pattern and moving to the top diffraction pattern. The shadow of the gold wire can clearly be seen. It is important to note that the distance from the wire does not influence the diffraction pattern. Electrons passing on the left side of the wire are deflected to a different

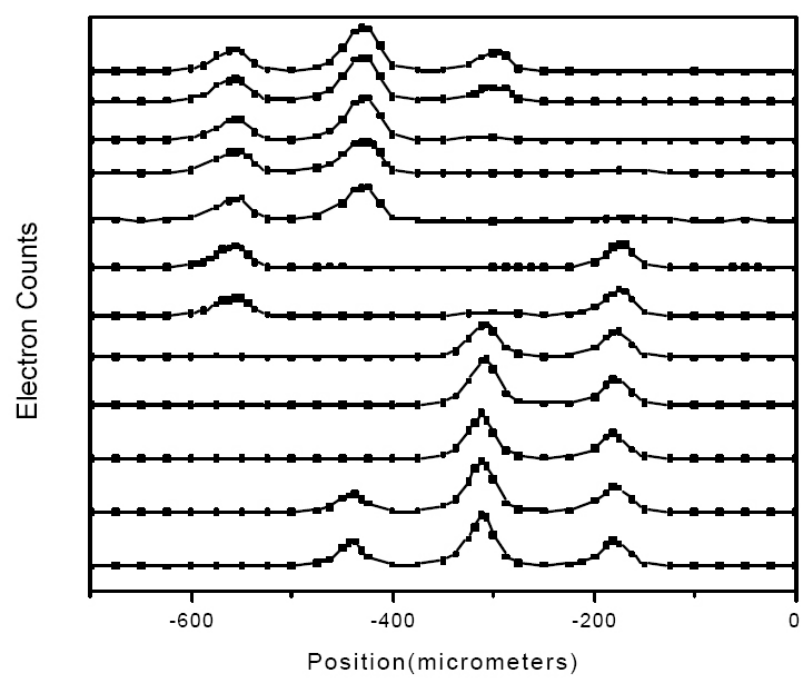

Figure 6: The working combination of a material grating and a biprism. See text for a description of the data.

position then electrons passing on the right side of the wire. This is in accordance with the constant phase gradients of a biprism and shows that combinations of material gratings and biprism are feasible. The middle pattern shows two diffraction peaks. This is effectively a large angle coherent beam-splitter. The angle between the outgoing beams is increased three times as compared to the angle between the adjacent diffraction orders emerging from the grating itself.

\section{Acknowledgements}

We would like to thank Tim Savas, Hank Smith and Paul Burrow for many helpful discussions. This work has been funded by NSF, the Research Corporation, and DOD-EPSCoR. 


\section{References}

[1] D.L. Freimund, K. Aflatooni, and H. Batelaan, Nature 413: 142 (2001).

[2] A. Tonomura, Electron Holography, Springer-Verlag, Berlin (1999).

[3] P. Dahl, Introduction to Electron and Ion Optics, Academic Press, New York (1973).

[4] G. Möllenstedt, H. Düker, Z. Phys. 145: 377 (1956).

[5] A.V. Crewe, D.N. Eggenberger, D.N. Wall, L.M. Welter, Rev. Sci. Instrum. 39: 576 (1968).

[6] R. Gupta, J.J. McClelland, Z.J. Jabbour, and R.J. Celotta, Appl. Phys. Lett. 67: 1,378 (1995).

[7] R.B. Doak, R.E. Grisenti, S. Rehbein, et al. Phys. Rev. Lett. 83: 4,229 (1999).

[8] T. Pfau, C. Kurtsiefer, C.S. Adams, et al., Phys. Rev. Lett. 71: 3,427 (1993).

[9] S. Nowak, N. Stuhler, T. Pfau, et al., Phys. Rev. Lett. 81: 5,792 (1998).

[10] D. Kleppner, Phys. Today 50, Part 1: 11 (1997).

[11] J.P. Dowling, J. GeaBanacloche, Adv. Atom Mol. Opt. Phys. 37: 1 (1996).

[12] T. Sleator, T. Pfau, V. Balykin, et al., Phys. Rev. Lett. 68: 1,996 (1992).

[13] M.J. Renn, D. Montgomery, O. Vdovin, D.Z. Anderson, C.E. Wieman, and E.A. Cornell, Phys. Rev. Lett. 75: 3,253 (1995).

[14] S. Bernet, R. Abfalterer, C. Keller, et al., Phys. Rev. A 62: 023606 (2000).

[15] P.L. Gould, G.A. Ruff, and D.E. Pritchard, Phys. Rev. Lett. 56: 827 (1986).

[16] C. Jönsson, D. Brandt, and S. Hirsch, Am. J. Phys. $42: 4$ (1974).

[17] P.L. Kapitza and P.A.M. Dirac, Proc. Camb. Phil. Soc. 29: 297 (1933).

[18] P.W. Milonni and J.H. Eberly, Lasers, John Wiley \& Sons, New York (1988).

[19] P.J. Martin, B.G. Oldaker, A.H. Miklich, and D.E. Pritchard, Phys. Rev. Lett. 60: 515, (1988).

[20] D. Freimund and H. Batelaan, submitted to Phys. Rev. Lett.

[21] E. Rasel, M.K. Oberthaler, H. Batelaan, J. Schmeidmayer, and A. Zeilinger, Phys. Rev. Lett. 75: 2,633 (1995).

[22] D.M. Giltner, R.W. McGowan, and S.A. Lee, Phys. Rev. Lett. 75: 2638 (1995). 